\title{
The YAG Lidar System Applied in LHAASO
}

\section{Q.N. Sun, ${ }^{1, *}$ L.S. Geng, ${ }^{2}$ X. Li, ${ }^{1}$ L. Chen, ${ }^{1}$ H. Liu, ${ }^{1}$ Y. Wang, ${ }^{1}$ F.R. Zhu ${ }^{1}$ and Y. Zhang ${ }^{2}$ on behalf of the LHAASO Collaboration}

(a complete list of authors can be found at the end of the proceedings)

\author{
${ }^{1}$ School of Physical Science and Technology School of Information Science and Technology, Southwest \\ Jiaotong University, 610031 Chengdu, Sichuan, China \\ ${ }^{2}$ Key Laboratory of Particle Astrophyics Experimental Physics Division Computing Center, Institute of \\ High Energy Physics, Chinese Academy of Sciences, 100049 Beijing, China \\ E-mail: longchen@swjtu.edu.cn
}

The atmospheric quality plays an important role in the air shower observation by the Wide Field-of-view Cherenkov Telescope Array ( WFCTA ) of LHAASO. A YAG imaging lidar system was developed to continuously monitor the calorimetric information. The accuracy of atmospheric monitoring is dependent on the pulse energy, the YAG laser's beam parameters and the angular repeatability of a High-precision 3D lifting Rotating Platform (HiRoP). Therefore, we designed an optical system for this lidar with a beam splitter to divide the laser beam into a reference beam and a calibrating beam with a certain ratio and coupled the beam paths with the movement of HiRoP. Thus, every pulse energy of the calibrating beam, which has the same energy fluctuation with respect to the reference beam recorded by a power meter, could be calculated by the ratio of the two beams. Great cares were also taken to characterize the beam size, polarization and divergence of the laser. Meanwhile, a high-precision home-made thermotank was designed to control the temperature and humidity to improve the performance and stability of our laser system, which resulting in a thermal fluctuation less than $2{ }^{\circ} \mathrm{C}$ inside the container in the winter at an altitude of $4410 \mathrm{~m}$. As a result, the pulse energy fluctuation of the laser beam for calibration was improved from $5 \%$ to less than $2 \%$. As a result, we have successfully attained distinguishable full-WFCTA-view scanning Laser images in different air conditions, which could be used for the atmospheric quality analysis in further.

Keywords: YAG-Lidar system, atmosphere monitoring, LHAASO

$37^{\text {th }}$ International Cosmic Ray Conference (ICRC 2021)

July 12th - 23rd, 2021

Online - Berlin, Germany

\footnotetext{
${ }^{*}$ Presenter
} 


\section{Introduction}

The Large High Altitude Air Shower Observatory ( LHAASO ) consists of three major components, $1 \mathrm{~km}^{2}$ Extensive Air Shower ( EAS ) array ( KM2A ), $78000 \mathrm{~m}^{2}$ water Cherenkov detector array ( WCDA ) and Wide Field of view Cerenkov Telescope Array ( WFCTA ). The LHAASO site is located in Haizi Mountain with an altitude of $4410 \mathrm{~m}$ in Daocheng, Sichuan. The main scientific goals of LHAASO project are the study of $40 \mathrm{GeV}-1 \mathrm{PeV}$ gamma ray astronomy and $10 \mathrm{TeV}-1$ $\mathrm{EeV}$ cosmic ray physics. The main scientific goal of WFCTA is to study the $10 \mathrm{TeV}-1 \mathrm{EeV}$ cosmic ray physics[1]. The WFCTA detect EAS makes use of the atmosphere as a giant calorimeter whose properties must be continuously monitored to ensure a reliable energy estimate. Atmospheric parameters influence both the production of Cherenkov light and its attenuation towards the WFCTA. The calibration system for the WFCTA consists of a series of devices, mainly including a YAG laser, two nitrogen lasers and two cloud cameras. In Figure 1, a picture of YAG Laser is shown. During WFCTA data acquisition, the LIDARs continuously scan the view of WFCTA and detect clouds and aerosols by analyzing the back scatter signal of a $355 \mathrm{~nm}$ pulsed laser beam. The cloud cameras use passive measurements of the infrared light and provide a picture of the whole sky every 5 minutes. The energy fluctuation of YAG Laser is $5 \%$ at $23{ }^{\circ} \mathrm{C}$. Every shot YAG Laser was divided into two beams by a BS spectroscope, calibration beam and reference, and the reference beam was monitored during the observation. The YAG Laser worked in a home-made thermotank with temperature fluctuation less than $2{ }^{\circ} \mathrm{C}$. And then ,the energy fluctuation of the calibration beam was improved to less than $2 \%$.

In this paper, we will describe the main characters of YAG Laser, the design and operation of YAG-Lidar and performance of Laser energy monitoring. In Sec. 2, we will discuss the setup, design and operation of YAG Lidar system. Data analysis and results follow in Sec. 3.

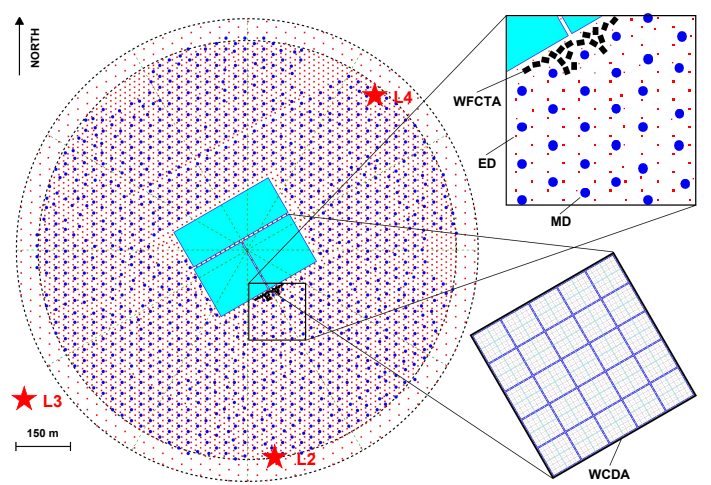

(a) The LHAASO layout.

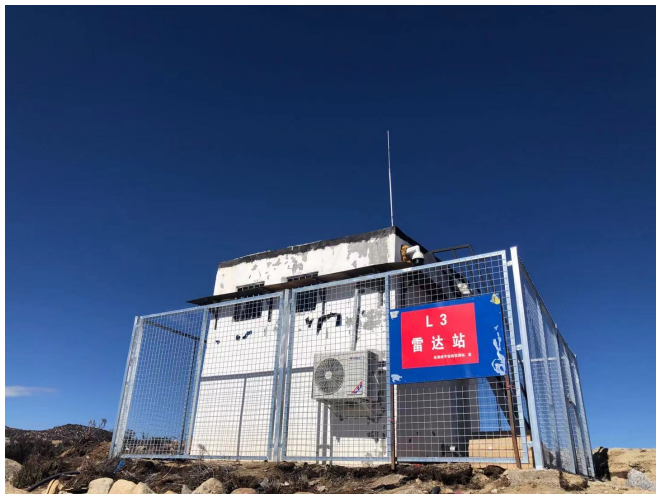

(b) Outdoor schematic diagram of YAG Lidar site.

Figure 1: a The LHAASO layout. The position of WFCTA, WCDA and KM2A is shown, red stars marks the position of Lidar facilities ( L2, L3, L4 ). b Outdoor schematic diagram of YAG Lidar site ( L3 ). 


\section{YAG-Lidar system}

YAG Lidar system consists of a YAG Laser, HiRoP with optical path, thermotank, Programmable Laser Controller ( PLC ) and external trigger system with GPS. Remote controlling system is implemented for operation of Laser and HiRoP.

During the data collection of WFCTA, the Calibration Laser scans the view of WFCTA with a set of azimuths and elevations to monitor atmospheric transparency and responses of telescopes [4]. The design of YAG optical path uses a set of high reflective mirrors to guide the light path coupling with the HiRoP, a wave plate was set to depolarize the beam and a beam spliter to draw a reference beam ( Ref-LASER ), used to monitor the energy of each outgoing laser ( Cali-LASER ) during laser emission. The schematic diagram of YAG Lidar inside the site is shown in Figure 2 below [2].

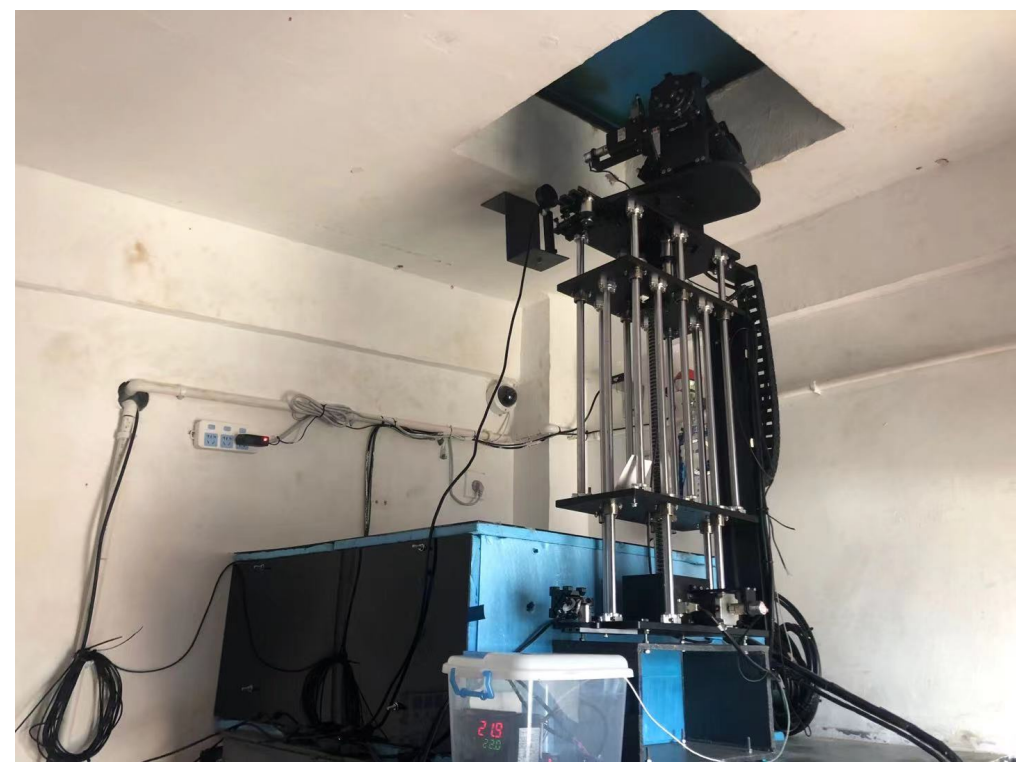

Figure 2: The schematic diagram of YAG Lidar inside the site, the black tank is the theromtank, YAG Laser and thermotank is located on an optic platform, the HiRop is in the middle of the picture, mirrors and energy detector is coupled on the HiRop.

\subsection{Design of optical path}

Coupling the optical path with the HiRoP [2], the "reflector-turntable" method is adopted for optical path design, so that the laser beam can be rotated with the HiRoP [2] to scan the whole view of WFCTA and the prototype, The schematic diagram of optical path is shown in Figure 3. The movements of the HiRoP involves lifting $(510 \mathrm{~mm})$, azimuth $360^{\circ}$, and tilting $180^{\circ}$. Specific methods to set mirrors was designed for the different movement modes of the HiRoP [5].

When the HiRoP goes upward and downward, the light between $M_{3}-M_{4}$ is required to coincide with the lifting direction of the HiRoP. A digital image processing method was introduced to solve the issue. Elevating and lowering the azimuth turntable, Laser beam could focus on the calibration 
screen, then the Laser spots is located by a digital camera, adjust the $M_{5}$ to coincide the spots at different height to determine optical path between $M_{3}$ and $M_{4}$. A digital camera is fixed over the center of azimuth turntable to locate the circle center of the azimuth turntable at different height, then $M_{5}$ could be adjusted to guide the light passing through the centers, then the optical path between $M_{5}$ and $M_{6}$ is determined. As for the optical path between $M_{6}$ and $M_{7}$, it is pointing to the north, which is determined by the sunshade directly calibration. A wave plate is used to depolarize the beam. The final polarization of the cal-beam before depolarization is $91.12 \%$ of the circular polarization [5].

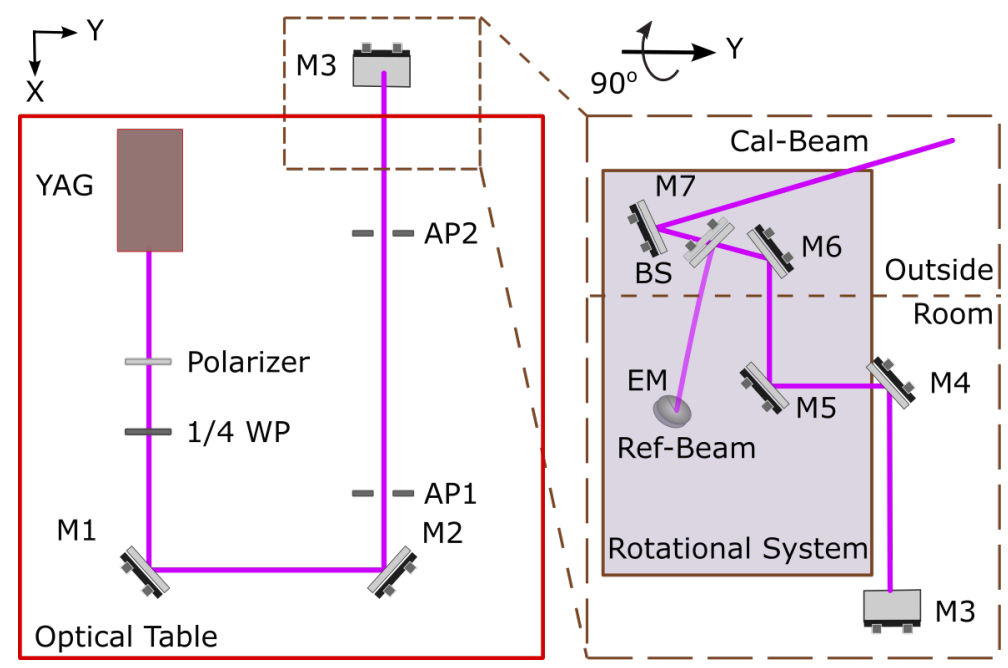

Figure 3: The design of optical path in YAG-Lidar system, the left part marked by a red line is the part in the thermotank on a optical platform, the right part $\left(M_{3}-M_{7}\right)$ is the part coupling with the HiRoP.

\subsection{Design of thermotank}

The thremotank's temperature control unit consists of heating device, feedback temperature probe, Proportion Integration Differentiation ( PID ) control device and voltage regulator. The cavity of thermotank is made of polystyrene foam board with a thickness of $2 \mathrm{~cm}$. The PID control device get immediately temperature by probe with frequency of $20 \mathrm{hz}$ and output current to the voltage regulator which powers the heating device to control the temperature inside. Considering the altitude of LHAASO is $4410 \mathrm{~m}$ and the heat dissipation efficiency, the temperature diff inside and outside the thermotank must larger than $5{ }^{\circ} \mathrm{C}$ to keep the temperature inside stable at $22{ }^{\circ} \mathrm{C}$ with a fluctuation less than $1{ }^{\circ} \mathrm{C}$ as shown in Figure 4 ( c ). The operation and accuracy measurement of Laser needs a stable working environment, the energy of YAG Laser is sensitive to temperature, energy changes $4.5 \% /{ }^{\circ} \mathrm{C}$ in the temperature range from $-5{ }^{\circ} \mathrm{C}$ to $15{ }^{\circ} \mathrm{C}$ as shown in Figure 4 ( b ). At the LHAASO experiment site, temperature changes from $11.5^{\circ} \mathrm{C}$ to $-31.8^{\circ} \mathrm{C}$ during operation of WFCTA in 2019 as shown in Figure 4 (a), a theromtank is designed to operate YAG Laser in a thermal fluctuation less than $2{ }^{\circ} \mathrm{C}$. 


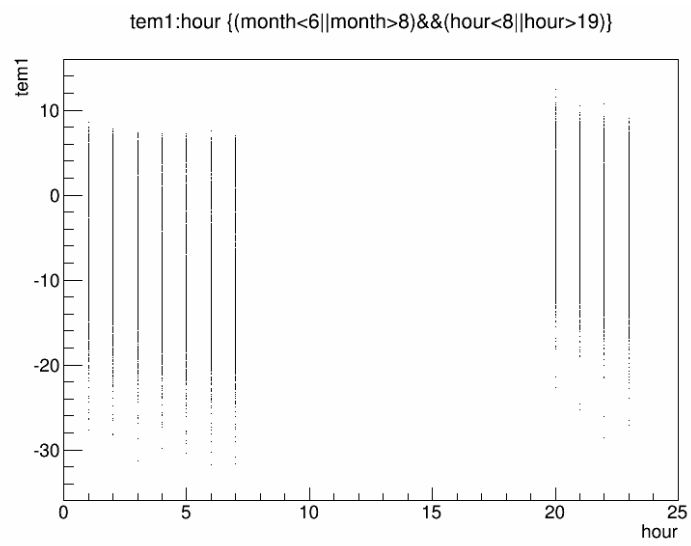

(a) Outdoor temperature on Haizi mountain.

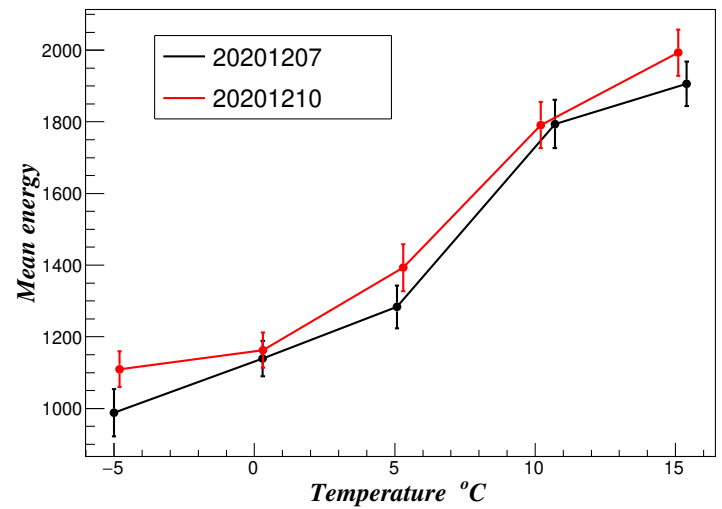

(b) The shotting energy of YAG Laser changes with temperature.

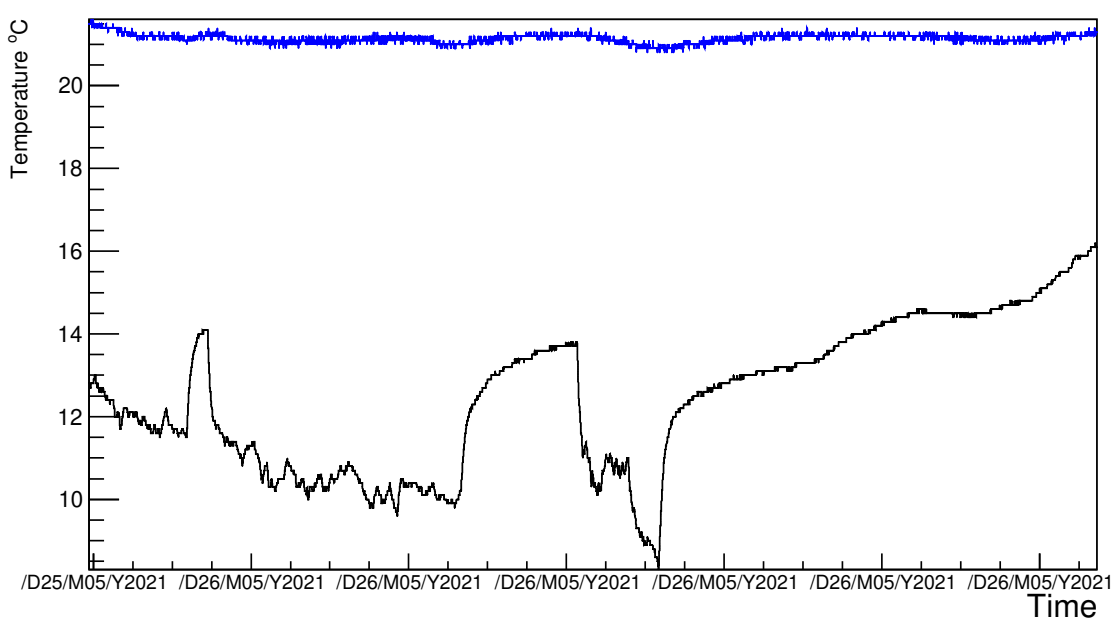

(c) Performance of thermotank.

Figure 4: a is the temperature of Haizi mountain at 2019, the WFCTA operate from 19 o'clock the night before to 8 o'clock the next day. $b$ is energy of YAG Laser changes with temperature, the red line and black line is result at different time. $\mathrm{c}$ is The temperature inside theromtank and the environment temperature changes at range from $8{ }^{\circ} \mathrm{C}$ to $16^{\circ} \mathrm{C}$., the blue line is the temperature inside thermotank, the black line is the temperature outside.

\section{Laser energy monitoring}

The fluctuation of YAG Laser is $5 \%$, to achieve the accuracy of calibration, every shot of Ref-LASER and Cali-LASER is collected by energy detector separately at same time. Marking Laser energy with GPS time, every shot is recorded and recognized. The stability of ratio is crucial to the accuracy of our calibration, it is affected by the fluctuation of the temperature inside the thermotank, the stability of optic elements applied in the Laser path and et c., fluctuation of ratio is less than $2 \%$ through the whole night as shown in Figure 5. 


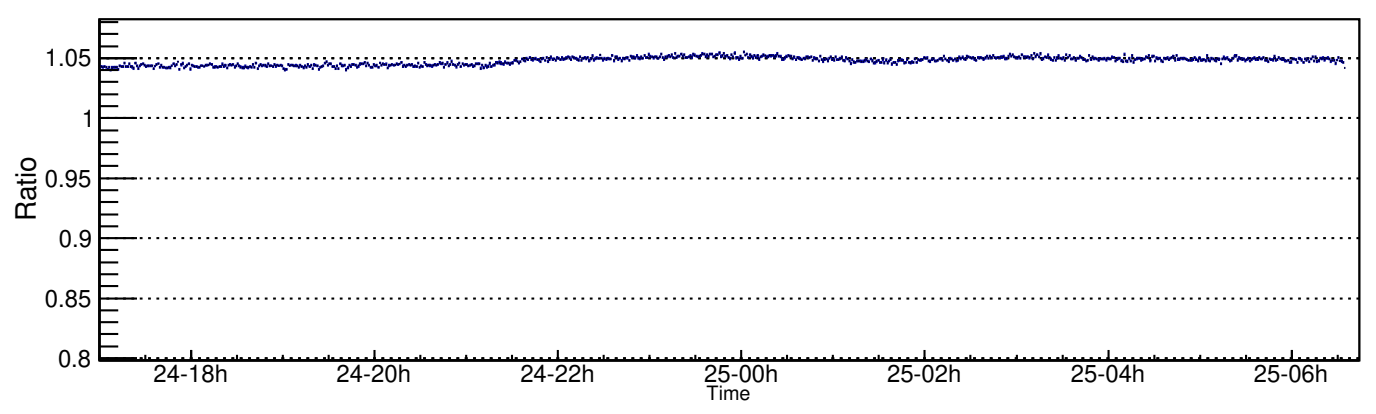

Figure 5: The energy ratio of Ref-LASER and Cali-LASER at the whole night, fluctuation of ratio is less than $2 \%$.

\section{Acknowledgement}

This work is supported in China by the Fundamental Research Funds for the Central Universities ( grant numbers 2682020CX77, 2682020CX73, 2682020CX74 ). It is also supported by the Science and Technology Department of Sichuan Province ( grant numbers 2021YFSY0031, 2020 YFSY0016 ), and by NSFC ( grant number 11947404 ), and by National Key R\&D program of China (grant number 2018YFA0404201).

\section{References}

[1] H. He (for LHAASO Coll.), Radiation Detection Technology and Methods 2-7, (2018)

[2] P., Roberts, M., Sommers, P., Travnicek, P., Valore, L.,Wiencke, L. (2005). The central laser facility at the Pierre Auger Observatory. 29th International Cosmic Ray Conference, ICRC $2005,8,335-338$.

[3] Nugent Paul W,Shaw Joseph A,Piazzolla Sabino. Infrared cloud imaging in support of Earthspace optical communication.[J]. Optics express,2009,17(10).

[4] G. Mie, Contributions to the optics of turbid media, particularly of colloidal metal solutions, Ann. Phys 25 (3) (1908) 377-445.

[5] Qiteng Zhao,Caixia Wu,Man Li. Design of Optical Path Switching Device Based on ElectroOptical Crystal[J]. Journal of Simulation,2021,9(2).

[6] Asamoah Benjamin O,Partanen Henri,Mohamed Sughra,Heikkinen Janne,Halder Atri,Koivurova Matias,Nečada Marek,Setälä Tero,Turunen Jari,Friberg Ari T,Hakala Tommi K. Polarization dependent beaming properties of a plasmonic lattice laser[J]. New Journal of Physics,2021,23(6). 


\section{Full Authors List: LHAASO Collaboration}

Zhen $\mathrm{Cao}^{1,2,3}$, F. Aharonian ${ }^{4,5}$, Q. An ${ }^{6,7}$, Axikegu ${ }^{8}$, L.X. Bai ${ }^{9}$, Y.X. Bai ${ }^{1,3}$, L.X. Bai ${ }^{9}$, Y.X. Bai ${ }^{1,3}$, Y.W. Bao ${ }^{10}$, D. Bastieri ${ }^{11}$, X.J. Bi ${ }^{1,2,3}$, Y.J. Bi ${ }^{1,3}$, H. Cai ${ }^{12}$, J.T. Cai ${ }^{11}$, Zhe Cao ${ }^{6,7}$, J. Chang ${ }^{13}$, J.F. Chang ${ }^{1,3,6}$, B.M. Chen ${ }^{14}$, E.S. Chen ${ }^{1,2,3}$, J. Chen ${ }^{9}$, Liang Chen $^{1,2,3}$, Liang Chen ${ }^{15}$, Long Chen ${ }^{8}$, M.J. Chen ${ }^{1,3}$, M.L. Chen ${ }^{1,3,6}$, Q.H. Chen ${ }^{8}$, S.H. Chen ${ }^{1,2,3}$, S.Z. Chen ${ }^{1,3}$, T.L. Chen ${ }^{16}$,X.L. $\mathrm{Chen}^{1,2,3}$, Y. Chen $^{10}$, N. Cheng ${ }^{1,3}$, Y.D. Cheng ${ }^{1,3}$, S.W. Cui ${ }^{14}$, X.H. Cuii ${ }^{17}$, Y.D. Cui ${ }^{18}$, B. D'Ettorre Piazzoli ${ }^{19}$, B.Z. Dai ${ }^{20}$, H.L. Dai $^{1,3,6}$, Z.G. Dai ${ }^{7}$, Danzengluobu ${ }^{16}$, D. della Volpe ${ }^{21}$, X.J. Dong ${ }^{1,3}$, K.K. Duan ${ }^{13}$, J.H. Fan ${ }^{11}$, Y.Z. Fan ${ }^{13}$, Z.X. Fan ${ }^{1,3}$, J. Fang ${ }^{20}$, K. Fang $^{1,3}$, C.F. Feng ${ }^{22}$, L. Feng ${ }^{13}$, S.H. Feng ${ }^{1,3}$, Y.L. Feng ${ }^{13}$, B. Gao ${ }^{1,3}$, C.D. Gao ${ }^{22}$, L.Q. Gao ${ }^{1,2,3}$, Q. Gao ${ }^{16}$, W. Gao ${ }^{22}$, M.M. Ge $^{20}$, L.S. Geng ${ }^{1,3}$, G.H. Gong ${ }^{23}$, Q.B. Gou ${ }^{1,3}$, M.H. Gu ${ }^{1,3,6}$, F.L. Guo ${ }^{15}$, J.G. Guo ${ }^{1,2,3}$, X.L. Guo ${ }^{8}$, Y.Q. Guo ${ }^{1,3}$, Y.Y. Guo ${ }^{1,2,3,13}$, Y.A. $\mathrm{Han}^{24}$, H.H. He $\mathrm{H}^{1,2,3}$, H.N. He ${ }^{13}$, J.C. He ${ }^{1,2,3}$, S.L. He ${ }^{11}$, X.B. He ${ }^{18}$, Y. He ${ }^{8}$, M. Heller ${ }^{21}$, Y.K. Hor ${ }^{18}$, C. Hou ${ }^{1,3}$, H.B. Hu ${ }^{1,2,3}$, S. $\mathrm{Hu}^{9}$, S.C. $\mathrm{Hu}^{1,2,3}$, X.J. $\mathrm{Hu}^{23}$, D.H. Huang ${ }^{8}$, Q.L. Huang ${ }^{1,3}$, W.H. Huang ${ }^{22}$, X.T. Huang ${ }^{22}$, X.Y. Huang ${ }^{13}$, Z.C. Huang ${ }^{8}$, F. Ji ${ }^{1,3}$, X.L. $\mathrm{Ji}^{1,3,6}$, H.Y. Jia ${ }^{8}$, K. Jiang ${ }^{6,7}$, Z.J. Jiang ${ }^{20}$, C. Jin ${ }^{1,2,3}$, T. Ke ${ }^{1,3}$, D. Kuleshov ${ }^{25}$, K. Levochkin ${ }^{25}$, B.B. Li ${ }^{14}$, Cheng Li ${ }^{6,7}$, Cong Li ${ }^{1,3}$,

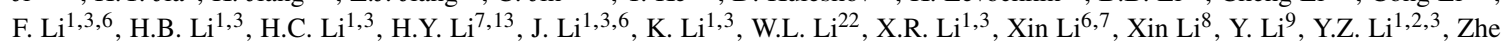

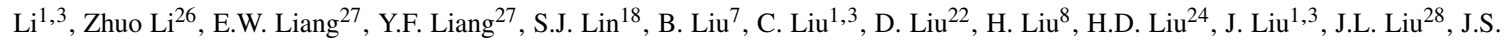

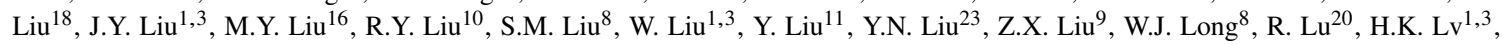

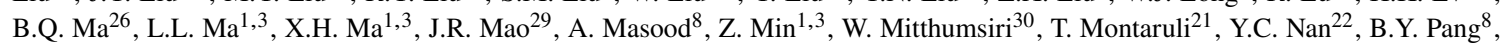
P. Pattarakijwanich ${ }^{30}$, Z.Y. Pei ${ }^{11}$, M.Y. Qi ${ }^{1,3}$, Y.Q. Qi ${ }^{14}$, B.Q. Qiao ${ }^{1,3}$, J.J. Qin ${ }^{7}$, D. Ruffolo ${ }^{30}$, V. Rulev ${ }^{25}$, A. Sáiz ${ }^{30}$, L. Shao ${ }^{14}$, O. Shchegolev $^{25,31}$, X.D. Sheng ${ }^{1,3}$, J.Y. Shi ${ }^{1,3}$, H.C. Song ${ }^{26}$, Yu.V. Stenkin ${ }^{25,31}$, V. Stepanov ${ }^{25}$, Y. Su ${ }^{32}$, Q.N. Sun ${ }^{8}$, X.N. Sun ${ }^{27}$, Z.B.

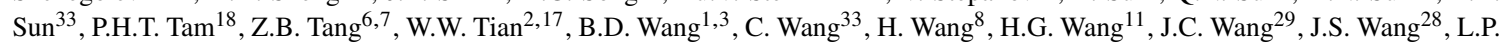
Wang $^{22}$, L.Y. Wang ${ }^{1,3}$, R.N. Wang ${ }^{8}$, W. Wang ${ }^{18}$, W. Wang ${ }^{12}$, X.G. Wang ${ }^{27}$, X.J. Wang ${ }^{1,3}$, X.Y. Wang ${ }^{10}$, Y. Wang ${ }^{8}$, Y.D. Wang ${ }^{1,3}$, Y.J. Wang $^{1,3}$, Y.P. Wang ${ }^{1,2,3}$, Z.H. Wang ${ }^{9}$, Z.X. Wang ${ }^{20}$, Zhen Wang ${ }^{28}$, Zheng Wang ${ }^{1,3,6}$, D.M. Wei $^{13}$, J.J. Wei ${ }^{13}$, Y.J. Wei ${ }^{1,2,3}$, T. Wen ${ }^{20}$, C.Y. $\mathrm{Wu}^{1,3}$, H.R. $\mathrm{Wu}^{1,3}$, S. Wu ${ }^{1,3}$, W.X. Wu ${ }^{8}$, X.F. Wu ${ }^{13}$, S.Q. Xi ${ }^{1,3}$, J. Xia ${ }^{7}, 13$, J.J. Xia ${ }^{8}$, G.M. Xiang ${ }^{2,15}$, D.X. Xiao ${ }^{16}$, G. Xiao ${ }^{1,3}$, H.B. Xiao ${ }^{11}$, G.G. Xin ${ }^{12}$, Y.L. Xin ${ }^{8}$, Y. Xing ${ }^{15}$, D.L. Xu ${ }^{28}$, R.X. Xu ${ }^{26}$, L. Xue ${ }^{22}$, D.H. Yan ${ }^{29}$, J.Z. Yan ${ }^{13}$, C.W. Yang 9 , F.F. Yang ${ }^{1,3,6}$, J.Y. Yang ${ }^{18}$, L.L. Yang ${ }^{18}$, M.J. Yang ${ }^{1,3}$, R.Z. Yang ${ }^{7}$, S.B. Yang ${ }^{20}$, Y.H. Yao ${ }^{9}$, Z.G. Yao ${ }^{1,3}$, Y.M. Ye ${ }^{23}$, L.Q. Yin ${ }^{1,3}$, N. Yin ${ }^{22}$, X.H. You $^{1,3}$, Z.Y. You ${ }^{1,2,3}$, Y.H. Yu ${ }^{22}$, Q. Yuan ${ }^{13}$, H.D. Zeng ${ }^{13}$, T.X. Zeng ${ }^{1,3,6}$, W. Zeng ${ }^{20}$, Z.K. Zeng ${ }^{1,2,3}$, M. Zha ${ }^{1,3}$, X.X. Zhai ${ }^{1,3}$, B.B. Zhang $^{10}$, H.M. Zhang ${ }^{10}$, H.Y. Zhang ${ }^{22}$, J.L. Zhang ${ }^{17}$, J.W. Zhang ${ }^{9}$, L.X. Zhang ${ }^{11}$, Li Zhang ${ }^{20}$, Lu Zhang ${ }^{14}$, P.F. Zhang ${ }^{20}$, P.P. Zhang ${ }^{14}$, R. Zhang ${ }^{7,13}$, S.R. Zhang ${ }^{14}$, S.S. Zhang ${ }^{1,3}$, X. Zhang ${ }^{10}$, X.P. Zhang ${ }^{1,3}$, Y.F. Zhang ${ }^{8}$, Y.L. Zhang ${ }^{1,3}$, Yi Zhang ${ }^{1,13}$, Yong Zhang ${ }^{1,3}$, B. $Z_{\text {Zhao }}^{8}$, J. Zhao ${ }^{1,3}$, L. Zhao ${ }^{6,7}$, L.Z. Zhao ${ }^{14}$, S.P. Zhao ${ }^{13,22}$, F. Zheng ${ }^{33}$, Y. Zheng ${ }^{8}$, B. Zhou ${ }^{1,3}$, H. Zhou ${ }^{28}$, J.N. Zhou ${ }^{15}$, P. Zhou ${ }^{10}$, R. Zhou $^{9}$, X.X. Zhou ${ }^{8}$, C.G. Zhu ${ }^{22}$, F.R. Zhu ${ }^{8}$, H. Zhu ${ }^{17}$, K.J. Zhu ${ }^{1,2,3,6}$ and X. Zuo ${ }^{1,3}$

${ }^{1}$ Key Laboratory of Particle Astrophyics \& Experimental Physics Division \& Computing Center, Institute of High Energy Physics, Chinese Academy of Sciences, 100049 Beijing, China.

${ }^{2}$ University of Chinese Academy of Sciences, 100049 Beijing, China.

${ }^{3}$ TIANFU Cosmic Ray Research Center, Chengdu, Sichuan, China.

${ }^{4}$ Dublin Institute for Advanced Studies, 31 Fitzwilliam Place, 2 Dublin, Ireland.

${ }^{5}$ Max-Planck-Institut for Nuclear Physics, P.O. Box 103980, 69029 Heidelberg, Germany.

${ }^{6}$ State Key Laboratory of Particle Detection and Electronics, China.

${ }^{7}$ University of Science and Technology of China, 230026 Hefei, Anhui, China.

${ }^{8}$ School of Physical Science and Technology \& School of Information Science and Technology, Southwest Jiaotong University, 610031 Chengdu, Sichuan, China.

${ }^{9}$ College of Physics, Sichuan University, 610065 Chengdu, Sichuan, China.

${ }^{10}$ School of Astronomy and Space Science, Nanjing University, 210023 Nanjing, Jiangsu, China.

${ }^{11}$ Center for Astrophysics, Guangzhou University, 510006 Guangzhou, Guangdong, China.

${ }^{12}$ School of Physics and Technology, Wuhan University, 430072 Wuhan, Hubei, China.

${ }^{13}$ Key Laboratory of Dark Matter and Space Astronomy, Purple Mountain Observatory, Chinese Academy of Sciences, 210023 Nanjing, Jiangsu, China.

${ }^{14}$ Hebei Normal University, 050024 Shijiazhuang, Hebei, China.

${ }^{15}$ Key Laboratory for Research in Galaxies and Cosmology, Shanghai Astronomical Observatory, Chinese Academy of Sciences, 200030 Shanghai, China.

${ }^{16}$ Key Laboratory of Cosmic Rays (Tibet University), Ministry of Education, 850000 Lhasa, Tibet, China.

${ }^{17}$ National Astronomical Observatories, Chinese Academy of Sciences, 100101 Beijing, China.

${ }^{18}$ School of Physics and Astronomy \& School of Physics (Guangzhou), Sun Yat-sen University, 519000 Zhuhai, Guangdong, China.

${ }^{19}$ Dipartimento di Fisica dell'Università di Napoli 'Federico II', Complesso Universitario di Monte Sant’Angelo, via Cinthia, 80126 Napoli, Italy.

${ }^{20}$ School of Physics and Astronomy, Yunnan University, 650091 Kunming, Yunnan, China.

${ }^{21}$ D'epartement de Physique Nucl'eaire et Corpusculaire, Facult'e de Sciences, Universit'e de Gen 'eve, 24 Quai Ernest Ansermet, 1211 Geneva, Switzerland.

${ }^{22}$ Institute of Frontier and Interdisciplinary Science, Shandong University, 266237 Qingdao, Shandong, China.

${ }^{23}$ Department of Engineering Physics, Tsinghua University, 100084 Beijing, China.

${ }^{24}$ School of Physics and Microelectronics, Zhengzhou University, 450001 Zhengzhou, Henan, China.

${ }^{25}$ Institute for Nuclear Research of Russian Academy of Sciences, 117312 Moscow, Russia. 
${ }^{26}$ School of Physics, Peking University, 100871 Beijing, China.

${ }^{27}$ School of Physical Science and Technology, Guangxi University, 530004 Nanning, Guangxi, China.

${ }^{28}$ Tsung-Dao Lee Institute \& School of Physics and Astronomy, Shanghai Jiao Tong University, 200240 Shanghai, China.

${ }^{29}$ Yunnan Observatories, Chinese Academy of Sciences, 650216 Kunming, Yunnan, China.

${ }^{30}$ Department of Physics, Faculty of Science, Mahidol University, 10400 Bangkok, Thailand.

${ }^{31}$ Moscow Institute of Physics and Technology, 141700 Moscow, Russia.

${ }^{32}$ Key Laboratory of Radio Astronomy, Purple Mountain Observatory, Chinese Academy of Sciences, 210023 Nanjing, Jiangsu, China.

${ }^{33}$ National Space Science Center, Chinese Academy of Sciences, 100190 Beijing, China. 